\title{
Exchange interaction of magnetic impurities in graphene
}

\author{
V. K. Dugaev ${ }^{1,2,3}$, V. I. Litvinov ${ }^{4}$, J. Barnaś ${ }^{5,6}$ \\ ${ }^{1}$ Department of Mathematics and Applied Physics, University of Technology, \\ Al. Powstańców Warszawy 6, 35-959 Rzeszów, Poland \\ ${ }^{2}$ Departamento de Física and CFIF, Instituto Superior Técnico, Av. Rovisco Pais, 1049-001 Lisbon, Portugal \\ ${ }^{3}$ Frantsevich Institute for Problems of Materials Science, \\ National Academy of Sciences of Ukraine, Vilde 5, 58001 Chernovtsy, Ukraine \\ ${ }^{4}$ WaveBand/Sierra Nevada Corporation, 15245 Alton Parkway, Suite 100, Irvine, CA 92618, USA \\ ${ }^{5}$ Department of Physics, Adam Mickiewicz University, Umultowska 85, 61-614 Poznań, Poland and \\ ${ }^{6}$ Institute of Molecular Physics, Polish Academy of Sciences, Smoluchowskiego 17, 60-179 Poznań, Poland
}

(Dated: September 5, 2018)

\begin{abstract}
The indirect exchange interaction between magnetic impurities localized in a graphene plane is considered theoretically, with the influence of intrinsic spin-orbit interaction taken into account. Such an interaction gives rise to an energy gap at the Fermi level, which makes the usual RKKY model not applicable. The results show that the effective indirect exchange interaction is described by a range function which decays exponentially with the distance between magnetic moments. The interaction is also shown to depend on whether the two localized moments belong to the same sublattice or are located in two different sublattices.
\end{abstract}

PACS numbers: 75.70.Ak,75.30.Et,75.30.Hx

\section{INTRODUCTION}

Physical properties of two-dimensional (2D) graphene planes are a subject of current interest due to their unique transport properties $\frac{1,2.3 .4}{1}$ Some of these properties originate from peculiarities of the corresponding electronic structure, or more specifically from the peculiar Fermi surface of a 2D graphene plane, which consists of six points located at the corners of the corresponding hexagonal 2D reciprocal lattice $\frac{5.6}{5}$ Only two of these points, however, are nonequivalent. Apart from this, the low energy excitations at these points can be approximated by the energy dispersion relation linear in the wavevector. Such electron states can be described by the Dirac equation (therefore, the corresponding points of the Brillouin zone are also termed as the Dirac points). All these features of the electronic structure make graphene very attractive from both theoretical and experimental points of view. This is because graphene is an ideal natural system for testing various theoretical models of $2 \mathrm{D}$ electron transport, including for instance the quantum Hall effect, or the effects due to the geometrical Berry phase 1.2

As concerns magnetic properties of graphene, these are still a subject of discussion in the relevant literature. Some experiments indicate on the existence of a spontaneous magnetic moment of the graphene planes ${ }^{7}$ However, physical origin of the spontaneous magnetization is not well understood. Several distinctly different mechanisms leading to ferromagnetism of graphene have been proposed, including the instability of the paramagnetic phase due to electron-electron interaction,,$\underline{8}$ indirect RKKY exchange coupling between magnetic moments of structural defects via mobile electrons, $\stackrel{9}{\underline{9}}$ and others ${ }^{10}$

The indirect exchange interaction between local magnetic moments is generally determined by electron excitations near the Fermi energy. Assuming that the
Fermi momentum in a graphene plane is zero and the excitations are gapless, a non-oscillatory indirect exchange interaction of the RKKY type has been found in Ref. 9]. Moreover, the corresponding exchange integral was found to be ferromagnetic, with the range function decaying as $R^{-3}$ with the distance $R$ between magnetic moments. However, the intrinsic spin-orbit (SO) interaction in graphene has been neglected in Ref. [9]. Such an interaction in graphene opens an energy gap at the Fermi level, 13 and makes the usual RKKY mechanism not relevant due to the absence of electrons at the Fermi level. Therefore, another mechanism of the indirect interaction between magnetic moments of structural defects should be developed.

In this paper we reconsider the indirect exchange interaction in graphene, taking into account the role of intrinsic SO interaction. If the SO gap is relatively small, the excitations across the gap contribute to the indirect exchange interaction $\stackrel{11.12}{ }$ We show that the exchange coupling is ferromagnetic, and the corresponding range function decays exponentially with the distance between magnetic moments - provided the chemical potential lies in the gap. Moreover, the spin-spin coupling is shown to be described approximately by the isotropic threedimensional (3D) Heisenberg Hamiltonian. Apart from this, the interaction is shown to be dependent on the location of magnetic impurities - the interaction for impurities located in the same sublattice is different from that between impurities located in different sublattices.

\section{MODEL AND EXCHANGE INTERACTION}

Indirect exchange interaction between magnetic moments of structural defects (for instance impurities) is determined by the electronics structure of the relevant system. To describe electron states in a graphene plane, 
we assume the Hamiltonian of non-interacting electrons in the following form ${ }^{13}$ :

$$
H=\hbar v\left(\tau_{x} p_{z} k_{x}+\tau_{y} k_{y}\right)+\Delta \tau_{z} p_{z} \sigma_{z},
$$

where three types of the Pauli matrices; $\boldsymbol{\tau}, \mathbf{p}$, and $\boldsymbol{\sigma}$, have been introduced. These matrices operate in different spaces, so their products in Eq.(1) should be understood as the direct matrix product. The matrix $\boldsymbol{\tau}$ acts in the (real) space of two nonequivalent sublattices. The matrix $\mathbf{p}$, in turn, acts in the (reciprocal) space of two nonequivalent Dirac points, whereas the matrix $\boldsymbol{\sigma}$ operates in the electron spin space. Finally, the constants $v$ and $\Delta$ in Eq.(1) are the velocity of electrons near the Fermi level (in the limit of no intrinsic SO interaction), and the parameter of SO interaction, respectively.

As we have already mentioned above, the Hamiltonian (1) operates in the 8-dimensional space, and describes electrons in the vicinity of two nonequivalent Dirac points located at the corners of the Brillouin zone, $\mathbf{k}_{0}$ and $-\mathbf{k}_{0}$, with $\mathbf{k}_{0}=\left(2 \pi / 3 a_{0}, 0\right)$ and $a$ denoting the lattice constant. The wavevector $k$ in Eq. 1 is measured from the location of a given Dirac point.

The energy spectrum of the Hamiltonian (1) consists of four-fold degenerate dispersion curves given by

$$
\varepsilon_{k}= \pm\left(\Delta^{2}+\hbar^{2} v^{2} k^{2}\right)^{1 / 2} .
$$

As follows from Eq. (2), the SO interaction opens a gap in the electronic states around the Fermi level, which extends from $-\Delta$ to $\Delta$.

We assume that the interaction of $2 \mathrm{D}$ electrons with magnetic impurities located at $\mathbf{R}_{i}(i=1,2, \ldots)$ in the graphene plane can be described by the following Hamiltonian:

$$
H_{i}=\frac{g_{0}}{n}\left[1+(-1)^{s} \tau_{z}\right]\left(\mathbf{S}_{i} \cdot \boldsymbol{\sigma}\right) \delta\left(\mathbf{r}-\mathbf{R}_{i}\right),
$$

where $\mathbf{S}_{i}$ is the spin moment of $i$-th impurity, $g_{0}$ is the parameter of exchange coupling between the localized spin and band electrons, and $n$ is the areal density of the host atoms in the graphene plane. The parameter $s=0,1$ distinguishes the two (A and $\mathrm{B}$ ) sublattices - if a given impurity belongs to the sublattice A (B) then $s=0(s=1)$. This form of interaction accounts for the exchange coupling that is sensitive to the location of the magnetic impurities in the two sublattices. However, the interaction (3) does not take into account the intervalley transitions. We consider first this simplified magnetic interaction, assuming that the rate of intervalley transitions is small. Later on we will briefly analyze the effect of intervalley scattering on the indirect exchange interaction.

The Green function corresponding to the Hamiltonian (1) has the form

$$
G_{\varepsilon}(\mathbf{k})=\frac{\varepsilon+\hbar v\left(\tau_{x} p_{z} k_{x}+\tau_{y} k_{y}\right)+\Delta \tau_{z} p_{z} \sigma_{z}}{[\varepsilon+i \delta \operatorname{sgn}(\varepsilon)]^{2}-\varepsilon_{k}^{2}} .
$$

The corresponding Green function in the energycoordinate representation can be derived from the above equation by integrating $G_{\varepsilon}(\mathbf{k})$ over the momentum in the vicinity of each valley,

$$
G_{\varepsilon}( \pm \mathbf{R})=\int \frac{d^{2} \mathbf{k}}{(2 \pi)^{2}} e^{ \pm i\left(t \mathbf{k}_{0}+\mathbf{k}\right) \cdot \mathbf{R}} G_{\varepsilon}(\mathbf{k})
$$

where $t= \pm \mathbf{1}$ corresponds to the two valleys located at $\pm \mathbf{k}_{\mathbf{0}}$, respectively.

Taking into account Eqs. (4),(5) and following Ref. 14] one obtains

$$
\begin{gathered}
G_{\varepsilon}( \pm \mathbf{R})=e^{ \pm i t \mathbf{k}_{0} \cdot \mathbf{R}}\left[-\frac{i\left(\varepsilon+\Delta \tau_{z} p_{z} \sigma_{z}\right)}{4 \hbar^{2} v^{2}}\right. \\
\times H_{0}^{(1)}\left(\frac{R \sqrt{\varepsilon^{2}-\Delta^{2}}}{\hbar v}\right) \pm \frac{\sqrt{\varepsilon^{2}-\Delta^{2}}}{4 \hbar^{2} v^{2} R}\left(R_{x} \tau_{x} p_{z}+R_{y} \tau_{y}\right) \\
\left.\times H_{1}^{(1)}\left(\frac{R \sqrt{\varepsilon^{2}-\Delta^{2}}}{\hbar v}\right)\right],
\end{gathered}
$$

where $H_{\nu}^{(1)}(z)$ are the Hankel functions which decay exponentially in the upper half-plane of the complex argument $z: \frac{15}{15}$ The Green function defined by Eq. (5) is the analytical function in the complex plane of $\varepsilon$, except for two cuts along the real axis from $-\infty$ to $-\Delta$ and from $\Delta$ to $+\infty$.

In a general case, the indirect exchange interaction is mediated by inter- and intra-valley electron excitations. As already mentioned above, we consider first the intravalley contribution and then discuss the role of intervalley transitions. In the loop approximation ${ }^{14.16}$ we find the interaction energy of two magnetic impurities $\mathbf{S}_{1}$ and $\mathbf{S}_{2}$ separated by a distance $R$ in the form

$$
E_{\text {int }}(R)=w_{\alpha \beta}^{s s^{\prime}}(R) S_{1 \alpha} S_{2 \beta},
$$

where

$$
\begin{aligned}
w_{\alpha \beta}^{s s^{\prime}}(R)=-\frac{i g_{0}^{2}}{n^{2}} \operatorname{Tr} & \int \frac{d \varepsilon}{2 \pi}\left[1+(-1)^{s} \tau_{z}\right] \sigma_{\alpha} G_{\varepsilon}(\mathbf{R}) \\
& \times\left[1+(-1)^{s^{\prime}} \tau_{z}\right] \sigma_{\beta} G_{\varepsilon}(-\mathbf{R}) .
\end{aligned}
$$

Making use of Eqs. (5) and (7) and calculating the trace, we find

$$
\begin{gathered}
w_{\alpha \beta}^{s s^{\prime}}(R)=\frac{i g_{0}^{2}}{\pi n^{2} \hbar^{4} v^{4}} \int_{i \delta}^{\infty+i \delta} d \varepsilon\left\{\delta _ { s s ^ { \prime } } \left[\left(\varepsilon^{2}-\Delta^{2}\right) \delta_{\alpha \beta}\right.\right. \\
\left.+\Delta^{2} \delta_{\alpha z} \delta_{\beta z}\right]\left[H_{0}^{(1)}\left(\frac{R \sqrt{\varepsilon^{2}-\Delta^{2}}}{v \hbar}\right)\right]^{2}+\delta_{\alpha \beta}\left(1-\delta_{s s^{\prime}}\right) \\
\left.\times\left(\varepsilon^{2}-\Delta^{2}\right)\left[H_{1}^{(1)}\left(\frac{R \sqrt{\varepsilon^{2}-\Delta^{2}}}{\hbar v}\right)\right]^{2}\right\}
\end{gathered}
$$

where the integral is along the real axis above the cut. After rotating the integration contour to the complex $\varepsilon$ plane along the imaginary axis, as explained in Ref. [14], 
we find from Eq. (9)

$$
\begin{gathered}
w_{\alpha \beta}^{s s^{\prime}}(R)=-\frac{4 g_{0}^{2}}{\pi^{3} n^{2} \hbar^{4} v^{4}} \int_{0}^{\infty} d \xi\left\{\delta_{s s^{\prime}}\right. \\
\times K_{0}^{2}\left(\frac{R \sqrt{\xi^{2}+\Delta^{2}}}{\hbar v}\right)\left[\left(\xi^{2}+\Delta^{2}\right) \delta_{\alpha \beta}-\Delta^{2} \delta_{\alpha z} \delta_{\beta z}\right] \\
\left.+\delta_{\alpha \beta}\left(1-\delta_{s s^{\prime}}\right)\left(\xi^{2}+\Delta^{2}\right) K_{1}^{2}\left(\frac{R \sqrt{\xi^{2}+\Delta^{2}}}{\hbar v}\right)\right\},
\end{gathered}
$$

where $K_{\nu}(z)$ are the MacDonald functions related to $H_{\nu}^{(1)}(z)$ by 15

$$
K_{\nu}(z)=\frac{i \pi}{2} e^{i \pi \nu / 2} H_{\nu}^{(1)}\left(z e^{i \pi / 2}\right) .
$$

When the spin-orbit interaction is taken into account, $\Delta \neq 0$, the range function given by Eq. (10) scales with the characteristic length $R_{0}=\hbar v / \Delta$. The derived exchange interaction is generally anisotropic. The interaction between in-plane spin components $(i=x, y)$ is given as

$$
w_{i i}^{s s^{\prime}}(R)=-\frac{4 g_{0}^{2}}{\pi^{3} n^{2} \hbar v R_{0}^{3}}\left\{\delta_{s s^{\prime}} I_{0}(R)+\left(1-\delta_{s s^{\prime}}\right) I_{1}(R)\right\},
$$

where

$$
I_{0,1}(R)=\int_{0}^{\infty} d x\left(x^{2}+1\right) K_{0,1}^{2}\left(\frac{R \sqrt{x^{2}+1}}{R_{0}}\right) .
$$

On the other hand, the interaction of the out-of-plane spin components is expressed via the formula

$$
w_{z z}^{s s^{\prime}}(R)=-\frac{4 g_{0}^{2}}{\pi^{3} n^{2} \hbar v R_{0}^{3}}\left\{\delta_{s s^{\prime}} I_{2}(R)+\left(1-\delta_{s s^{\prime}}\right) I_{1}(R)\right\}
$$

with

$$
I_{2}(R)=\int_{0}^{\infty} d x x^{2} K_{0}^{2}\left(\frac{R \sqrt{x^{2}+1}}{R_{0}}\right) .
$$

The interaction described by Eqs. (12) and (14) is formally valid under the condition $R \geq a$, with $a$ being the lattice constant.

The asymptotics of MacDonald functions at large argument, $\frac{15}{5} K_{\nu}(z) \simeq \sqrt{\pi / 2 z} e^{-z}$, leads to the exponential decrease of interactions, $I_{0,1}(R) \sim$ $R^{-3 / 2} \exp \left(-2 R / R_{0}\right)$ and $I_{2}(R) \sim R^{-5 / 2} \exp \left(-2 R / R_{0}\right)$ for $R \gg R_{0}$. Thus, the value of $R_{0}$ determines an effective range of the exchange interaction.

The magnitude of the indirect exchange interaction depends on the relative position of the spins in the graphene sublattices. As mentioned above, the exchange interaction is described by an anisotropic Heisenberg model. However, $I_{0}(R) \approx I_{2}(R)$, so the anisotropy is weak and one may approximate the exchange interaction by a 3D-isotropic Heisenberg Hamiltonian, irrespective which sublattice the magnetic impurities belong to.

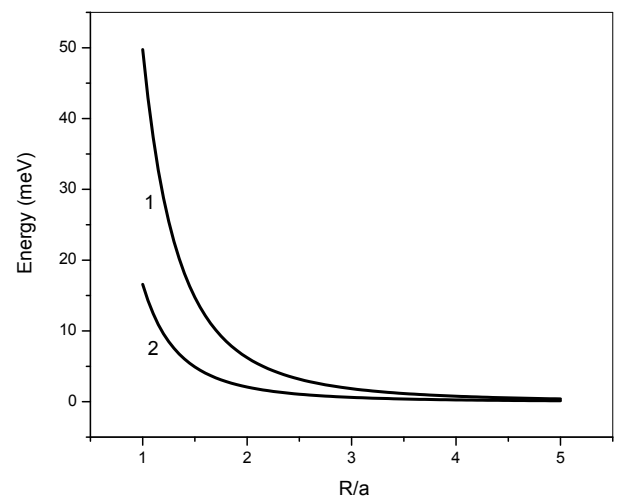

FIG. 1: Range functions in the presence of spin-orbit interaction. The curve labelled with 1 represents $\left|w_{i i}^{s s}(R)\right|$, whereas the curve labelled with 2 corresponds to $\left|w_{i i}^{s s}(R)\right|$.

As follows from Eq. (10), the gapless fermions $(\Delta=0)$ mediate the indirect exchange interaction that is also 3Disotropic, but with the power-law range function,

$$
w_{\alpha \beta}^{s s^{\prime}}(R)=-\frac{4 g_{0}^{2} \delta_{\alpha \beta}}{\pi^{3} n^{2} \hbar v R^{3}}\left\{F_{0} \delta_{s s^{\prime}}+F_{1}\left(1-\delta_{s s^{\prime}}\right)\right\}
$$

where

$$
\begin{aligned}
& F_{0}=\int_{0}^{\infty} x^{2} K_{0}^{2}(x) d x \approx 0.31 \\
& F_{1}=\int_{0}^{\infty} x^{2} K_{1}^{2}(x) d x \approx 0.93 .
\end{aligned}
$$

The interaction given by Eq. (16) is the term similar to that derived in Ref. [9]. It should be noted, however, that the gap due to spin-orbit interaction induces an exponential decay of the range function, even in samples with low density of non-magnetic impurities.

The indirect exchange interaction is ferromagnetic. The numerical results in Fig. 1 show the absolute value of the interaction energy when the interacting moments are in the same or different sublattices. In our estimations we used the carbon-carbon distance $a=1.42 \AA$, the Fermi velocity $\hbar v=5.7 \mathrm{eV} \AA, g_{0} \simeq 1 \mathrm{eV}, \underline{8.9}$ and $\Delta \simeq 1.2 \mathrm{~K} \stackrel{13}{S}$ From the unit cell area of $S=3 a^{2} \sqrt{3} / 2$ containing two carbon atoms, one can estimate the coupling coefficient $g_{0} / n \simeq 2.62 \mathrm{eV} \AA^{2}$. In order to illustrate the range function dependence on $R / a$, we took into account the scaling coefficient $a / R_{0}=2.5 \times 10^{-5}$.

The numerical results explicitly show the exponential decay of the range function with the distance between two magnetic impurities. Moreover, they also demonstrate that the coupling is stronger when the magnetic moments are localized on different sublattices. 


\section{MAGNETIC POLARIZATION DUE TO A SINGLE IMPURITY}

The exchange interaction between a magnetic impurity and $2 \mathrm{D}$ electrons gives rise to a spin polarization of the latter ones. The corresponding spin density distribution $\mathbf{M}(R)$ can be calculated as a response of the electron system to the perturbation due to a single magnetic impurity $\mathbf{S}_{0}$ at $\mathbf{R}=0$. As a result, one finds $\mathbf{M}(R)$ in the form

$$
\begin{array}{r}
\mathbf{M}(R)=-i g_{0} \operatorname{Tr} \int \frac{d \varepsilon}{2 \pi} \boldsymbol{\sigma} G_{\varepsilon}(\mathbf{R})\left(1+(-1)^{\tau} \tau_{z}\right) \\
\times\left(\boldsymbol{\sigma} \cdot \mathbf{S}_{0}\right) G_{\varepsilon}(-\mathbf{R}) .
\end{array}
$$

The assumed exchange interaction between a magnetic impurity and 2D electrons is local, see Eq. (3). As a result, the spin polarization at a given point of the lattice, mediated via the $2 \mathrm{D}$ electrons, is sensitive to the location of the magnetic impurity (in one sublattice or the other), see Eq. (16).

Performing calculations similar to those presented above one finds

$$
M_{\alpha}(R)=\frac{w_{\alpha \beta}^{s \neq s^{\prime}} S_{0 \beta}}{g_{0}} .
$$

The above result shows that spin polarization has no oscillatory component. Apart from this, one can conclude from Eqs. (10) and (19), that the $z$-component of magnetic density $\mathbf{M}(R)$ is nonzero only in the case when the magnetic impurity has a non-vanishing spin component perpendicular to the graphene plane.

\section{EFFECT OF INTERVALLEY TRANSITIONS}

As we have already mentioned above, graphene has two nonequivalent valleys in the Brillouin zone, which are associated with two Dirac points. Moreover, the exchange coupling between localized moment and 2D band electrons assumed in Eq. 3 does not allow for intervalley transitions. However, such transitions are allowed by symmetry of the system and should be taken into account. To do this we consider an additional term in the interaction between a localized moment and band electrons. This new term may be written in the form

$$
H_{i v}=\frac{g_{v}}{n} \sum_{i}\left(1+(-1)^{s} \tau_{z}\right) p_{x}\left(\mathbf{S}_{i} \cdot \boldsymbol{\sigma}\right) \delta\left(\mathbf{r}-\mathbf{R}_{i}\right),
$$

where $g_{v}$ is the corresponding coupling parameter.

The above form of interaction between 2D band electrons and localized moments gives rise to additional terms in the indirect exchange interaction. These terms are of oscillatory type, $\sim \cos \left(2 \mathbf{k}_{0} \cdot \mathbf{R}\right)$. However, we believe that the coupling coefficient $g_{v}$ is much smaller than $g_{0}$, so the main results and conclusions described above will not be changed by including the contribution following from the interaction (20).

\section{SUMMARY AND CONCLUSIONS}

In this paper we have calculated the indirect exchange interaction between two magnetic impurities localized in a graphene plane. The interaction is mediated by twodimensional electrons and holes, and acquires the form of an isotropic 3D Heisenberg coupling, with the coupling parameter being of ferromagnetic type. The exchange parameter has an exponentially decaying long distance tail determined by the spin-orbit interaction.

Thus, graphene doped with magnetic impurities may become ferromagnetic at a certain impurity concentration and below a certain temperature. Magnetic graphene could be then an ideal system for experimental investigations of 2D transport of spin polarized electrons. Moreover, one may also expect that magnetic graphene could be very useful in spintronics devices, where electron spin plays a role comparable to its charge 17

We have calculated the exchange interaction for $T=0$. Strictly speaking, our results refer to $T \ll \Delta$. The effect of temperature on the exchange interaction in narrowgap semiconductors has been studied by Rusin, 18 who showed that for the chemical potential in the gap, the temperature corrections do not change the main result: even for $T \gg \Delta$, the interaction at large distances is exponentially decaying.

When calculating the exchange interaction we assumed the chemical potential $\mu=0$. The results do not change for a finite $\mu$ as long as the chemical potential is located within the energy gap, which corresponds to the undoped material. In principle, the chemical potential in graphene can be varied either by a gate voltage or by doping with impurities. As a result, the chemical potential can be shifted into the conduction or valence bands, and the interaction between magnetic impurities includes then the usual RKKY exchange term via free carriers. As in conventional metals or doped semiconductors, this gives an additional oscillating term with the oscillation period determined by the Fermi momentum of electrons (holes). However, the mechanism of exchange interaction considered in this paper is still working for $|\mu|>\Delta$. The resulting interaction is then due to both the virtual transitions of electrons through the gap and the excitations of real electron-hole pairs in the vicinity of the Fermi energy.

Magnitude of SO interaction in graphene was recently a subject of extensive discussion in the relevant literature. ${ }^{19,20,21}$ A similar problem was also discussed in the case of carbon nanotubes. ${ }^{22,23,24}$ Some results indicate that the SO interaction in graphene can be very small. However, the key point is that this interaction is nonzero, which results in a finite exchange interaction length. As we have shown in this paper, the nonzero SO interaction leads to some anisotropy of the magnetic interaction between magnetic ions.

The energy gap in graphene can be also induced by excitonic effects $\stackrel{25.26}{ }$, thus being not related to the spin degrees of freedom. As a result, the exchange interaction mediated by virtual transitions through the excitonic gap 
$\Delta_{e x c}$ acquires the finite length $\hbar v / \Delta_{e x c}$. The magnetic interaction is then strictly isotropic.

\section{Acknowledgements}

We thank N. M. R. Peres for discussions. This research was supported by the Portuguese program POCI under Grant No. POCI/FIS/58746/2004, polish Ministry of Science and Higher Education through the Research Project Nr. N202 142 31/2598 (2006-2009), and by the STCU Grant No. 3098 in Ukraine.
${ }^{1}$ K. S. Novoselov, A.K. Geim, S. V. Morozov, D. Jiang, M. I. Katsnelson, I. V. Grigorieva, S. V. Dubonos, and A. A. Firsov, Nature 438, 197 (2005).

2 K. S. Novoselov, E. McCann, S. V. Morozov, V.I. Fal'ko, M. I. Katsnelson, U. Zeitler, D. Jiang, F. Schedin, and A.K. Geim, Nature Physics 2, 177 (2006).

3 J. Tworzydło, B. Trauzettel, M. Totov, A. Rycerz, and C.W.J. Beenakker, Phys. Rev. Lett. 96, 246802 (2006).

4 S.V. Morozov, K.S. Novoselov, M.I. Katsnelson, F. Schedin, L.A. Ponomarenko, D. Jiang, and A.K. Geim, Phys. Rev. Lett. 97, 16801 (2006).

5 D.P. DiVincenzo and E.J. Mele, Phys. Rev. B 29, 1685 (1984).

6 R. Saito, G. Dresselhaus, and M.S. Dresselhaus, Physical Properties of Carbon Nanotubes, Imperial College Press, 1998.

7 K. H. Han, D. Spemann, P. Esquinazi, R. Höhne, R. Riede, and T. Butz, Adv. Mater. 15, 1719 (2003).

8 N. M. R. Peres, F. Guinea, and A. H. Castro Neto, Phys. Rev. B 72, 174406 (2005).

9 M. A. H. Vozmediano, M. P. López-Sancho, T. Stauber, and F. Guinea, Phys. Rev. B 72, 155121 (2005).

10 K. Nomura and A.H. MacDonald, Phys. Rev. Lett. 96, 256602 (2006).

11 N. Bloembergen and T. Rowland, Phys. Rev. 97, 1697 (1955).

12 V. I. Litvinov and V. K. Dugaev, Phys. Rev. Lett. 86, 5593 (2001).

13 C. L. Kane and E. J. Mele, Phys. Rev. Lett. 95, 226801 (2005).
14 V. K. Dugaev and V. I. Litvinov, Superlattices Microstruct. 16, 413 (1994).

15 Handbook of Mathematical Functions, edited by $\mathrm{M}$. Abramowitz and I. I. Stegun (Nat. Bur. Stand. Appl. Math. Ser. 55, 1964).

16 V. I. Litvinov and V. K. Dugaev, Phys. Rev. B 58, 3584 (1998).

17 G. A. Prinz, Science 282, 1660 (1998); S. A. Wolf, D. D. Awschalom, R. A. Buhrman, J. M. Daughton, S. von Molnár, M. L. Roukes, A. Y. Chtchelkanova, and D. M. Treger, Science 294, 1488 (2001); I. Žutić, J. Fabian, and S. Das Sarma, Rev. Mod. Phys. 76, 323 (2004).

18 T. M. Rusin, Phys. Rev. B 53, 7217 (1996).

19 Y. Yao, F. Ye, X.-L. Qi, S.-C. Zhang, and Z. Fang, condmat/0606350.

${ }^{20}$ H. Min, J. E. Hill, N. A. Sinitsyn, B. R. Sahu, L. Kleinman, and A. H. MacDonald, condmat/0606504.

21 D. Huertas-Hernando, F. Guinea, and A. Brataas, condmat/0606580.

22 T. Ando, J. Phys. Soc. Jpn. 69, 1757 (2000).

23 A. De Martino, R. Egger, K. Hallberg, and C. A. Balseiro, Phys. Rev. Lett. 88, 206402 (2002).

24 L. Chico, M. P. López-Sancho, and M. C. Muñoz, Phys. Rev. Lett. 93, 176402 (2004).

25 D.V. Khveshchenko, Phys. Rev. Lett. 87, 206401 (2001); 87, 246802 (2001).

26 E. V. Gorbar, V. P. Gusynin, V. A. Miransky, and I. A. Shovkovy, Phys. Rev. B 66, 045108 (2002). 